\title{
Interaction corrections to the Hall coefficient at intermediate temperatures
}

\author{
Gábor Zala $^{(a, b)}$, B.N. Narozhny ${ }^{(a)}$, and I.L. Aleiner ${ }^{(a, b)}$ \\ ${ }^{(a)}$ Department of Physics and Astronomy, SUNY at Stony Brook, Stony Brook, NY, 11794, USA \\ (b) Physics Department, Lancaster University, LA1 4YB, Lancaster, UK
}

\begin{abstract}
We investigate the effect of electron-electron interaction on the temperature dependence of the Hall coefficient of $2 \mathrm{D}$ electron gas at arbitrary relation between the temperature $T$ and the elastic mean-free time $\tau$. At small temperature $T \tau \ll \hbar$ we reproduce the known relation between the logarithmic temperature dependences of the Hall coefficient and of the longitudinal conductivity. At higher temperatures, this relation is violated quite rapidly; correction to the Hall coefficient becomes $\propto 1 / T$ whereas the longitudinal conductivity becomes linear in temperature.
\end{abstract}

Introduction - It is well known that electron-electron interaction at low temperatures $(T \tau \ll \hbar)$ leads to the logarithmically divergent correction $\delta \sigma_{x x}$ to the longitudinal conductivity of $2 \mathrm{D}$ electron gas. Whereas for a wide range of temperatures the sign and the magnitude of those corrections are not universal (in particular due to the Fermi liquid type interactions in the triplet channel), one property remains intacte: there is no logarithmic interaction correction to the Hall conductivity $\sigma_{x y}$ for any type of the interaction. When the interaction correction is still smaller than the Drude conductivity, the latter fact can be also re-written as

$$
\gamma=2 \text {, }
$$

where the parameter $\gamma$ is defined as

$$
\gamma(T)=-\frac{\partial_{T} \ln \rho_{x y}(T)}{\partial_{T} \ln \sigma_{x x}(T)}
$$

with $\rho_{x y}$ being the Hall resistivity.

Equation (11) is not controlled by any symmetries of the system and comes about as a result of the diffusive approximation justified only for $T \tau \ll \hbar$. For the $2 \mathrm{D}$ electron gas based on semiconductor heterostructures 3 this condition is easily violated. Therefore, one can no longer rely on the relation (11) for $T \tau \gtrsim \hbar$. The theory describing the corresponding temperature behavior of $\gamma$ is still not available. Our goal in this paper is to derive the corresponding analytic formulas. Temperature dependence of the Hall coefficient much weaker than predicted by Eq. (2) was recently reported in Refl.

Method - We will use the kinetic equation formalism for the interaction corrections which we briefly summarize below and then apply it to the calculation of the Hall coefficient. The detailed derivation of the formalism and the region of its validity can be found in Ref. 5 .

The electric current is expressed in terms of the distribution function $f(t ; \epsilon, \vec{r}, \vec{n})$ as

$$
\vec{J}(t, \vec{r})=e \nu v_{F} \int_{-\infty}^{\infty} d \epsilon\langle\vec{n} f(t ; \epsilon, \vec{r}, \vec{n})\rangle_{n} .
$$

Here $\nu$ is the density of states of the noninteracting system taken at the Fermi surface and $v_{F}$ is the Fermi ve- locity, $\vec{n}=(\cos \theta, \sin \theta)$ is the unit vector in the direction of the electron momentum and angular averaging is $\langle\ldots\rangle_{n}=\int \frac{d \theta}{2 \pi} \ldots$. The Boltzmann-like equation for the stationary and homogeneous distribution function is

$$
\left[e v_{F}(\vec{n} \vec{E}) \frac{\partial}{\partial \epsilon}+\omega_{c} \vec{b}\left(\vec{n} \times \frac{\partial}{\partial \vec{n}}\right)\right] f=\operatorname{St}\{f\} .
$$

where $\vec{E}$ is the applied electric field and $\omega_{c}$ is the cyclotron frequency due to the external magnetic field and $\vec{b}$ is the unit vector along the field.

All of the interaction effects are taken into account in the elastic and inelastic parts of the collision integral, $\operatorname{St}\{f\}=\operatorname{St}_{e l}\{f\}+\operatorname{St}_{i n}\{f\}$. The inelastic part does not contribute to the Hall current. The relevant, elastic part can be written as (in all intermediate formulae we use the units with $\hbar=1$ )

$$
\begin{aligned}
& \mathrm{St}_{e l}\{f\}=-\frac{f(\epsilon, \vec{n})-\langle f(\epsilon, \vec{n})\rangle_{n}}{\tau} \\
& +I_{0}(\epsilon, \vec{n})\langle f(\epsilon, \vec{n})\rangle_{n}+n_{\alpha} I_{1}^{\alpha \beta}(\epsilon)\left\langle n_{\beta} f(\epsilon, \vec{n})\right\rangle_{n},
\end{aligned}
$$

where the second line describes the effect of interaction on elastic collisions and

$$
\begin{aligned}
& I_{0}(\epsilon, \vec{n})=-\frac{8}{\tau} \int \frac{d \omega}{2 \pi}\left\{n_{\alpha} K_{0}^{\alpha \beta}(\omega)\left\langle n_{\beta} f(\epsilon-\omega, \vec{n})\right\rangle_{n}\right. \\
& \left.+\frac{n_{\alpha} L_{0}^{\alpha \beta}(\omega)}{2} e E_{\beta} \frac{\partial}{\partial \epsilon}\langle f(\epsilon-\omega, \vec{n})\rangle_{n}\right\} \\
& I_{1}^{\alpha \beta}(\epsilon)=-\frac{8}{\tau} \int \frac{d \omega}{2 \pi} K_{1}^{\alpha \beta}(\omega)\langle f(\epsilon-\omega, \vec{n})\rangle_{n} .
\end{aligned}
$$

One can easily see that the elastic collision integral vanishes in equilibrium $f(\epsilon, \vec{n})=f(\epsilon), E=0$. The explicit definitions of $K_{0}, K_{1}$, and $L_{0}$ entering Eqs. (5) are:

$$
\begin{aligned}
& K_{1}^{\alpha \beta}(\omega)=\operatorname{Im} \int \frac{d^{2} q}{(2 \pi)^{2}} \mathcal{D}^{R}(\omega, \vec{q}) \\
& \quad \times\left\{\left\langle n_{\alpha} D\right\rangle\left\langle D n_{\beta}\right\rangle-\frac{\delta_{\alpha \beta}}{2}\left(\langle D\rangle\langle D\rangle+i \frac{\partial}{\partial \omega}\langle D\rangle\right)\right\}, \\
& K_{0}^{\alpha \beta}(\omega)=\operatorname{Im} \int \frac{d^{2} q}{(2 \pi)^{2}} \mathcal{D}^{R}(\omega, \vec{q})
\end{aligned}
$$




$$
\begin{aligned}
& \times\left\{\left\langle n_{\alpha} D n_{\beta}\right\rangle\langle D\rangle-\frac{i}{v_{F}} \frac{\partial}{\partial q_{\alpha}}\left\langle D n_{\beta}\right\rangle-\left\langle D n_{\alpha}\right\rangle\left\langle D n_{\beta}\right\rangle\right\}, \\
& L_{0}^{\alpha \beta}(\omega)=-\operatorname{Re} \int \frac{d^{2} q}{(2 \pi)^{2}} \mathcal{D}^{R}(\omega, \vec{q}) \\
& \quad \times\left\{\langle D\rangle \frac{\partial}{\partial q_{\beta}}\left\langle n_{\alpha} D\right\rangle-\left\langle D n_{\alpha}\right\rangle \frac{\partial}{\partial q_{\beta}}\langle D\rangle-\left\langle D n_{\alpha} \frac{\partial}{\partial q_{\beta}} D\right\rangle\right\} .
\end{aligned}
$$

Here, $\mathcal{D}^{R}$ denotes the retarded interaction propagator in singlet channe $\mathbb{E}$ and the angular averaging is

$$
\begin{aligned}
\langle a D b\rangle & \equiv \int \frac{d \theta d \theta^{\prime}}{(2 \pi)^{2}} a(\vec{n}) D\left(\vec{n}, \vec{n}^{\prime} ; \omega, \vec{q}\right) b\left(\vec{n}^{\prime}\right), \\
\langle a D b D c\rangle & \equiv \int \frac{d \theta d \theta^{\prime} d \theta^{\prime \prime}}{(2 \pi)^{3}} a(\vec{n}) D\left(\vec{n}, \vec{n}^{\prime}\right) b\left(\vec{n}^{\prime}\right) D\left(\vec{n}^{\prime}, \vec{n}^{\prime \prime}\right) c\left(\vec{n}^{\prime \prime}\right)
\end{aligned}
$$

for arbitrary functions $a, b$. The function $D\left(\vec{n}, \vec{n}^{\prime} ; \omega, \vec{q}\right)$ describes the classical motion of a particle on the energy shell $\epsilon_{F}$ in a magnetic field:

$$
\begin{aligned}
& {\left[-i \omega+i v_{F} \vec{n} \vec{q}+\omega_{c} \vec{b}\left(\vec{n} \times \frac{\partial}{\partial \vec{n}}\right)\right] D\left(\vec{n}, \vec{n}^{\prime}\right)} \\
& +\frac{1}{\tau}\left[D\left(\vec{n}, \vec{n}^{\prime}\right)-\left\langle D\left(\vec{n}, \vec{n}^{\prime}\right)\right\rangle_{n}\right]=2 \pi \delta\left(\theta-\theta^{\prime}\right) .
\end{aligned}
$$

It is noteworthy, that unlike in the ordinary Boltzmann equation, the Lorentz force affects also the collision processes as one can see from Eqs. (6) and (7).
The above equations are written for the interaction in the singlet channel only. In a situation where both triplet and singlet channels are present, but the distribution function does not have a spin structure (no Zeeman splitting or non-equilibrium spin occupation present), one has to replace in Eq. (6)

$$
\mathcal{D}^{R} \rightarrow \mathcal{D}_{s}^{R}+3 \mathcal{D}_{t}^{R}
$$

Calculation of the Hall coefficient - We calculate the Hall resistivity $\rho_{x y}$ in the first order in magnetic field. We notice that due to the rotational and reflection symmetries of the system

$$
K_{i}^{\alpha \beta}(\omega)=\delta_{\alpha \beta} K_{i}(\omega)+\epsilon_{\alpha \beta} \omega_{c} \tau \tilde{K}_{i}(\omega),
$$

and the same structure for the $L$ - kernel. Here $\epsilon_{\alpha \beta}$ is the antisymmetric tensor, $\epsilon_{x y}=1$.

In order to calculate the conductivity we look for a solution of Eqs. (4) - (5)

$$
f(\vec{n}, \epsilon)=f_{F}(\epsilon)+n_{\alpha} \Gamma_{\alpha}^{(1)}(\epsilon)+\left(\omega_{c} \tau\right) n_{\alpha} \epsilon_{\alpha \beta} \Gamma_{\beta}^{(2)}(\epsilon),
$$

where $f_{F}(\epsilon)=1 /\left(e^{\epsilon / T}+1\right)$ is the Fermi distribution function (all the energies are counted from the Fermi level), and the unknown quantities $\Gamma^{(1,2)}$ are first order in the electric field and zeroth order in the magnetic field.

Substituting Eq. (10) into Eqs. (44:5), we obtain the following system of equations:

$$
\begin{aligned}
& e v_{F} E_{\alpha} \frac{\partial f_{F}(\epsilon)}{\partial \epsilon}=-\frac{\Gamma_{\alpha}^{(1)}(\epsilon)}{\tau}-\frac{4}{\tau} \int \frac{d \omega}{2 \pi}\left[K_{1}(\omega) f_{F}(\epsilon-\omega) \Gamma_{\alpha}^{(1)}(\epsilon)+K_{0}(\omega) f_{F}(\epsilon) \Gamma_{\alpha}^{(1)}(\epsilon-\omega)\right] \\
& -\frac{4 f_{F}(\epsilon)}{\tau} \int \frac{d \omega}{2 \pi} L_{0}(\omega) e E_{\alpha} \frac{\partial}{\partial \epsilon} f_{F}(\epsilon-\omega), \\
& \Gamma_{\alpha}^{(1)}(\epsilon)=-\Gamma_{\alpha}^{(2)}(\epsilon)-4 \int \frac{d \omega}{2 \pi} f_{F}(\epsilon-\omega)\left[K_{1}(\omega) \Gamma_{\alpha}^{(2)}(\epsilon)+\tilde{K}_{1}(\omega) \Gamma_{\alpha}^{(1)}(\epsilon)\right] \\
& \left.-4 \int \frac{d \omega}{2 \pi} f_{F}(\epsilon)\left[K_{0}(\omega) \Gamma_{\alpha}^{(2)}(\epsilon-\omega)+\tilde{K}_{0}(\omega) \Gamma_{\alpha}^{(1)}(\epsilon-\omega)\right]-4 f_{F}(\epsilon) \int \frac{d \omega}{2 \pi} \frac{\partial}{\partial \omega_{c}} \tilde{L}_{(} \omega\right) e E_{\alpha} \frac{\partial}{\partial \epsilon} f_{F}(\epsilon-\omega) .
\end{aligned}
$$

We solve Eqs. 11a by iteration and obtain

$$
\begin{aligned}
\Gamma_{\alpha}^{(1)}(\epsilon)= & -e v_{F} \tau E_{\alpha} \frac{\partial f_{F}(\epsilon)}{\partial \epsilon}+4 e v_{F} \tau \int \frac{d \omega}{2 \pi}\left[K_{1}(\omega) f_{F}(\epsilon-\omega) \frac{\partial f_{F}(\epsilon)}{\partial \epsilon}+K_{0}(\omega) f_{F}(\epsilon) \frac{\partial f_{F}(\epsilon-\omega)}{\partial \epsilon}\right] E_{\alpha} \\
& -4 f_{F}(\epsilon) \int \frac{d \omega}{2 \pi} L_{0}(\omega) e E_{\alpha} \frac{\partial}{\partial \epsilon} f_{F}(\epsilon-\omega), \\
\Gamma_{\alpha}^{(2)}= & -\Gamma_{\alpha}^{(1)}-4 e v_{F} \tau \int \frac{d \omega}{2 \pi} f_{F}(\epsilon-\omega) \frac{\partial f_{F}(\epsilon)}{\partial \epsilon}\left[K_{1}(\omega)-\tilde{K}_{1}(\omega)\right] E_{\alpha} \\
& -4 e v_{F} \tau \int \frac{d \omega}{2 \pi} f_{F}(\epsilon) \frac{\partial f_{F}(\epsilon-\omega)}{\partial \epsilon}\left[K_{0}(\omega)-\tilde{K}_{0}(\omega)\right] E_{\alpha}-4 f_{F}(\epsilon) \int \frac{d \omega}{2 \pi} \tilde{L}_{0}(\omega) e E_{\alpha} \frac{\partial}{\partial \epsilon} f_{F}(\epsilon-\omega) .
\end{aligned}
$$

We substitute Eq. (12) into Eq. (10) $\left[\Gamma^{(1)}\right.$ does not contribute to the Hall current], and the result into Eq. (3). Performing angular averaging and integration over $\epsilon$ one finds components of the conductivity tensor.

Rather than writing explicit results for the Hall conductivity we present the expression for the Hall resistivity, $\rho_{x y}=\rho_{H}^{D}+\delta \rho_{x y}, \quad \frac{\delta \rho_{x y}}{\rho_{H}^{D}}=-\frac{\delta \sigma_{x y}}{\left(\omega_{c} \tau\right) \sigma_{D}}-2 \frac{\delta \sigma_{x x}}{\sigma_{D}}$, where $\sigma_{D}=e^{2} \nu v_{F}^{2} \tau / 2$ is the Drude conductivity, and $\rho_{H}^{D}$ is the classical Hall resistivity $\left(\rho_{H}^{D}=-\omega_{c} \tau / \sigma_{D}\right)$. We find 


$$
\frac{\delta \rho_{x y}}{\rho_{H}^{D}}=\int_{-\infty}^{\infty} \frac{d \omega}{\pi} \frac{\partial}{\partial \omega}\left(\omega \operatorname{coth} \frac{\omega}{2 T}\right)\left[\tilde{K}_{1}(\omega)-\tilde{K}_{0}(\omega)+\frac{\tilde{L}_{0}(\omega)}{v_{F} \tau}+\frac{L_{0}(\omega)}{v_{F} \tau}\right] .
$$

What remains is to find the explicit expressions for the kernels entering into Eq. (13). To do so, we solve Eq. (7) up to the first order in magnetic field:

$$
\begin{aligned}
& D\left(\vec{n}, \vec{n}^{\prime}\right)=D_{1}\left(\vec{n}, \vec{n}^{\prime}\right)-\omega_{c} \int \frac{d \theta_{1}}{2 \pi} D_{1}\left(\vec{n}, \vec{n}_{1}\right)\left(\vec{n}_{1} \times \frac{\partial}{\partial \vec{n}_{1}}\right) D_{1}\left(\vec{n}_{1}, \vec{n}^{\prime}\right), \\
& D_{1}\left(\vec{n}, \vec{n}^{\prime}\right)=2 \pi \delta\left(\theta-\theta^{\prime}\right) D_{0}(\vec{n})+D_{0}(\vec{n}) D_{0}\left(\vec{n}^{\prime}\right) \frac{C}{C \tau-1}, \\
& C=\sqrt{(-i \omega+1 / \tau)^{2}+v_{F}^{2} q^{2}}, \quad D_{0}(\vec{n})=\frac{1}{-i \omega+i v_{F} \vec{n} \vec{q}+1 / \tau} .
\end{aligned}
$$

We note, that only the second term on the R.H.S. of Eq. (14a) contributes to $\tilde{K}_{i}$ and $\tilde{L}_{0}$. Substituting Eqs. (14) into Eqs. (6), the resulting kernels into Eq. (13) and performing the angular integration we obtain the results for the correction to the diagonal conductivity, $\delta \sigma_{x x}$, and to the Hall resistivity,

$$
\left(\begin{array}{l}
\delta \rho_{x y} / \rho_{H}^{D} \\
\delta \sigma_{x x} / \sigma_{D}
\end{array}\right)=\operatorname{Im} \int_{-\infty}^{\infty} \frac{d \omega}{\pi} \frac{\partial}{\partial \omega}\left(\omega \operatorname{coth} \frac{\omega}{2 T}\right) \int \frac{q d q}{4 \pi} \mathcal{D}^{R}(\omega, q)\left(\begin{array}{c}
B_{x y}(\omega, q) \\
B_{x x}(\omega, q)
\end{array}\right)
$$

where the form-factors are defined as

$$
\begin{aligned}
& B_{x y}=-\left\{\frac{2 v_{F}^{2} q^{2} / \tau^{2}}{C^{3}(C-1 / \tau)^{3}}+\frac{v_{F}^{2} q^{2}[2 C-5(-i \omega+1 / \tau)]}{2 \tau^{2} C^{5}(C-1 / \tau)^{2}}+\frac{(-i \omega+1 / \tau)[C-(-i \omega+1 / \tau)]}{\tau^{2} C^{4}(C-1 / \tau)^{2}}\right\}, \\
& B_{x x}=\left\{\frac{v_{F}^{2} q^{2} / \tau^{2}}{C^{3}(C-1 / \tau)^{3}}+\frac{3 v_{F}^{2} q^{2}}{2 \tau C^{3}(C-1 / \tau)^{2}}+\frac{2[C-(-i \omega+1 / \tau)]}{C(C-1 / \tau)^{2}}+\frac{2 C-1 / \tau}{C v_{F}^{2} q^{2}}\left(\frac{C-(-i \omega+1 / \tau)}{C-1 / \tau}\right)^{2}\right\} .
\end{aligned}
$$

Expression for the form-factor $B_{x x}$ was obtained before in Ref. f and cited here for comparison. It is noteworthy that $B_{x y, x x}(\omega, q=0)=0$ as it is dictated by the gauge invariance.

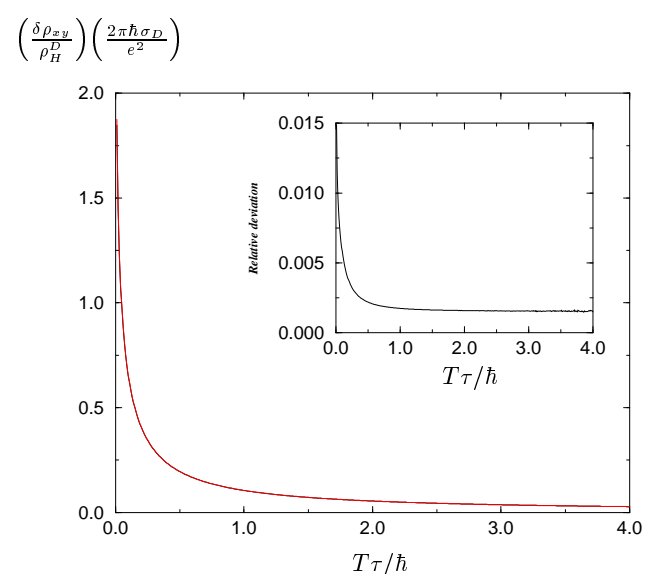

FIG. 1. Singlet channel correction to Hall resistivity $\delta \rho_{x y}^{\rho}$, given by Eq.(17) (solid line) and interpolation formula (18) (dashed line). On this scale two curves are indistinguishable, and the relative deviation is blown up on the inset.

Equations (16) enable one to anticipate the behavior of parameter $\gamma$ from Eq. (2) even before the form of the interaction in Eq. (15) is specified. At low temperatures, the integrals are determined by $\omega, q v_{F} \ll 1 / \tau$. In this case both formfactors in Eq. (16) are controlled by the first terms in the R.H.S. As a result, one arrives to Eq. (11). At larger $q \gg 1 / v_{F} \tau$, however, the be- havior of the formfactors is completely different, $B_{x x} \propto$ $1 / q^{2}, B_{x y} \propto 1 / q^{4}$, so the relation (11) can no longer hold.

To study the details of temperature behavior of $\gamma$, one needs to evaluate the integrals in Eq. (15) for both the singlet and the triplet channel propagator (see Eq. (50) and Eq. (60) in Ref. 5):

$$
\mathcal{D}_{t}^{R}=-\frac{1}{\nu} \frac{C-1 / \tau}{i \omega+\frac{F_{0}^{\sigma}+1}{F_{0}^{\sigma}}(C-1 / \tau)}
$$

where $F_{0}^{\sigma}$ is the interaction constant in the triplet channel, and singlet propagator, $\mathcal{D}_{s}^{R}$, is obtained by putting $F_{0}^{\sigma} \rightarrow \infty$. For completely spin-polarized electrons only the singlet channel correction is present. We find

$$
\begin{aligned}
& \frac{\delta \rho_{x y}^{\rho}}{\rho_{H}^{D}}=-\frac{e^{2}}{4 \pi^{2} \sigma_{D}}\left\{-4 \mathbf{C}+\frac{1}{24 \pi T \tau} \psi^{\prime}\left(1+\frac{1}{2 \pi T \tau}\right)\right. \\
& -\frac{11}{12} \psi\left(1+\frac{1}{2 \pi T \tau}\right)-\frac{19}{2} \int_{0}^{1} d x x \psi\left(1+\frac{x}{2 \pi T \tau}\right) \\
& \left.+5 \int_{0}^{1} d x x^{2} \psi\left(1+\frac{x}{2 \pi T \tau}\right)\right\},
\end{aligned}
$$

where $\mathbf{C} \approx 0.577$ is Euler's constant, $\psi(x)$ is the digamma function. Equation (17) can be approximated by the following interpolation formula 


$$
\frac{\delta \rho_{x y}^{\rho}}{\rho_{H}^{D}}=\frac{e^{2}}{\pi^{2} \hbar \sigma_{D}} \ln \left(1+\frac{11 \pi}{192} \frac{\hbar}{T \tau}\right) .
$$

This formula reproduces the logarithmic behavion in the diffusive limit $(T \tau \ll \hbar)$ in accordance with Eq. (1), and $\frac{\delta \rho_{x y}^{\rho}}{\rho_{H}^{D}}=\frac{e^{2}}{\pi^{2} \hbar \sigma_{D}} \frac{11 \pi}{192} \frac{\hbar}{T \tau}$, for $T \tau \gg \hbar$, in contrast with the linear dependence of the diagonal conductivity in this regime ${ }^{2}$. Finally, at intermediate temperatures Eq. (18) provides accurate (for numerical reasons) description of the crossover, see Fig. 1.

The triplet channel correction is calculated analogously and the interpolation formula similar to Eq. (18) reads

$$
\begin{gathered}
\frac{\delta \rho_{x y}^{\sigma}}{\rho_{H}^{D}}=\frac{3 e^{2}}{\pi^{2} \hbar \sigma_{D}}\left\{1-\frac{1}{F_{0}^{\sigma}} \ln \frac{1+F_{0}^{\sigma}+(T \tau / \hbar) g\left(F_{0}^{\sigma}\right)}{1+T \tau / \hbar}\right\} \times \\
\ln \left(1+\frac{11 \pi}{192} \frac{\hbar}{T \tau}\right) .
\end{gathered}
$$

Once again, Eq. (19) reproduces the asymptotic behavior at high and low temperature and gives numerically accurate results in the intermediate region. The definition of the smooth function $g(x)$ is

$$
\begin{gathered}
\ln g(x)=\frac{4}{11}\left[-5 f_{3}(x)-12 f_{2}(x)-3 f_{1}(x)+4 f_{0}(x)\right], \\
f_{j}(x)=\frac{1}{x^{j}}\left(\ln [1+x]+\sum_{n=1}^{j} \frac{(-x)^{n}}{n}\right),
\end{gathered}
$$

and it has the following asymptotic behavior, $g(x)=$ $1+x+\frac{3}{22} x^{2}+\ldots, x \ll 1$, and $g(x) \rightarrow e^{-70 / 33}, x \rightarrow-1$. The latter asymptotic is realized when the system is close to the Stoner instability.

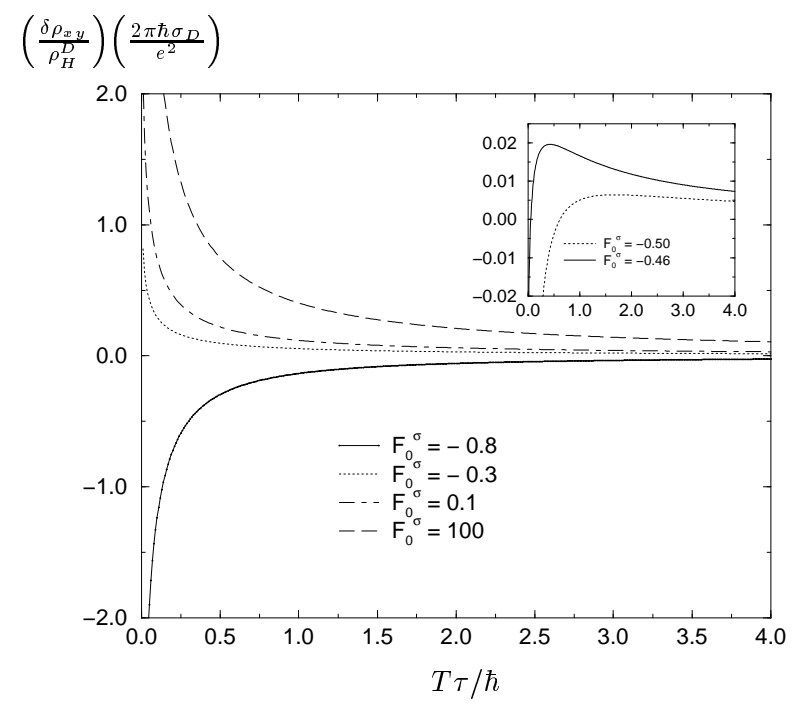

FIG. 2. Total correction to Hall resistivity $\delta \rho_{x y}$ for different values of the Fermi liquid parameter $F_{0}^{\sigma}$. For $-0.61<F_{0}^{\sigma}<-0.45$, the temperature dependence is non-monotonous, though very weak, see inset.

The total correction to the Hall coefficient, $\delta \rho_{x y}=$ $\delta \rho_{x y}^{\rho}+\delta \rho_{x y}^{\sigma}$, is shown on Fig. 2. Let us note in passing, that if the electron system is polarized by strong in-plane field, only the singlet correction (18) remains.

Finally, we present the temperature dependence of the quantity $\gamma$ from Eq.(2). The plot shown in Fig. 3 was obtained with the help of Eqs. (18), (19) of the present paper and Eq. (16) of Ref. 5 . We note, that the slope of this curve at zero temperature is always finite. According to the figure, $\gamma(T)$ 's deviation from the value of 2 happens already at much lower temperatures than expected: it changes by a factor of 2 at about $T \tau / \hbar \simeq 0.1$ for the weak coupling $\left(F_{0}^{\sigma} \ll 1\right)$ case.

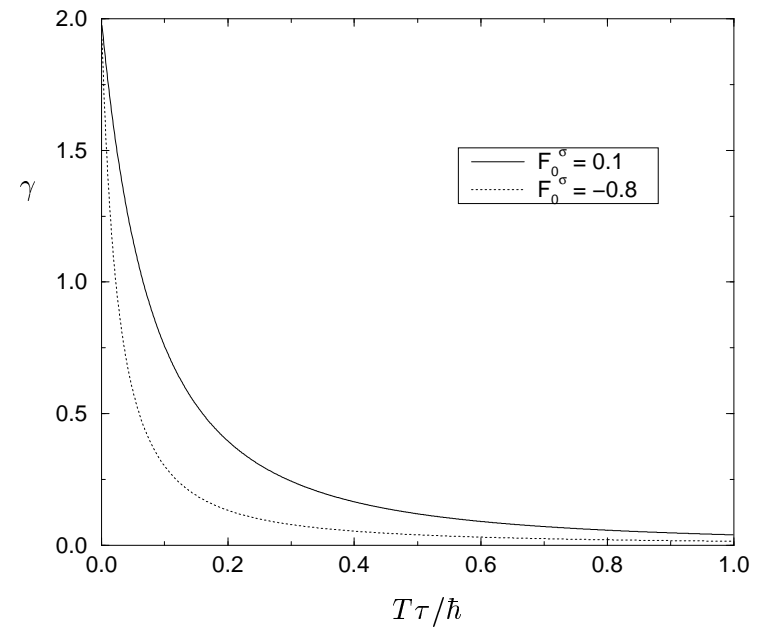

FIG. 3. Temperature dependence of the parameter $\gamma$, see Eq. (2), relating the Hall coefficient to $\sigma_{x x}$.

Summary - To conclude, we investigated the temperature dependence of the Hall resistivity of $2 \mathrm{D}$ electron gas for arbitrary values of $T \tau / \hbar$. It is shown that whereas the relation between the Hall and diagonal resistivity (1) indeed holds for $T \tau \ll \hbar$, it is rapidly violated at higher temperatures, see Fig. 3 .

Acknowledgments - We are grateful to B.L. Altshuler, S.V. Kravchenko, M.Yu. Reyzer, and A.K. Savchenko for stimulating discussions. One of us (I.A.) was supported by the Packard foundation. Work in Lancaster University was partially funded by EPSRC-GR/R01767.

${ }^{1}$ B.L. Altshuler and A.G. Aronov in Electron-Electron Interactions in Disordered Systems, eds. A.L. Efros, M. Pollak (North-Holland, Amsterdam, 1985).

2 B.L. Altshuler et. al., Phys. Rev. B 22, 5142 (1980).

${ }^{3}$ For a recent review and references see E. Abrahams, S. V. Kravchenko, and M. P. Sarachik, Rev. Mod. Phys. 73, 251 (2001).

${ }^{4}$ Y.Y. Proskuryakov et.al., Phys. Rev. Lett. 86, 4895 (2001).

${ }^{5}$ G. Zala, B.N. Narozhny, and I.L. Aleiner, condmat/0105406. 